\title{
Interrogating conserved elements of diseases using Boolean combinations of orthologous phenotypes
}

\author{
Woods, John O. ${ }^{* 1,2}$, Tien, Matthew Z. ${ }^{\dagger 1,3}$, and Marcotte, \\ Edward M..$^{\ddagger 1}$ \\ ${ }^{1}$ Center for Systems and Synthetic Biology, Institute for Cellular \\ and Molecular Biology, The University of Texas at Austin \\ ${ }^{2}$ Applied Space Exploration Laboratory, College of Engineering \\ and Mineral Resources, West Virginia University \\ ${ }^{3}$ Department of Biochemistry and Molecular Biology, Gordon \\ Center for Integrative Science, University of Chicago
}

April 11, 2015

\begin{abstract}
Conserved genetic programs often predate the homologous structures and phenotypes to which they give rise; eyes, for example, have evolved several dozen times, but their development seems to involve a common set of conserved genes. Recently, the concept of orthologous phenotypes (or phenologs) offered a quantitative way to describe this property. Phenologs are phenotypes or diseases from separate species who share an unexpectedly large set of their associated gene orthologs. It has been shown that the phenotype pairs which make up a phenolog are mutually predictive in terms of the genes involved. Recently, we demonstrated the ranking of gene-phenotype association predictions using multiple phenologs from an array of species. In this work, we demonstrate a computational method which provides a more targeted view of the conserved pathways which give rise to diseases. Our approach involves the generation of synthetic pseudophenotypes made up of Boolean combinations (union, intersection, and difference) of the gene sets for phenotypes from our database. We search for diseases that overlap significantly with these Boolean phenotypes, and find a number of highly predictive combinations. While set unions produce less specific predictions (as expected), intersection and differencebased combinations appear to offer insights into extremely specific aspects of target diseases. For example, breast cancer is predicted by zebrafish
\end{abstract}

\footnotetext{
*john.woods@mail.wvu.edu

†mtien@utexas.edu

‡marcotte@icmb.utexas.edu
} 
methylmercury response minus metal ion response, with predictions $M T$ COI, JUN, SOD2, GADD $45 B$, and BAX all involved in the pro-apoptotic response to reactive oxygen species, thought to be a key player in cancer. We also demonstrate predictions from Arabidopsis Boolean phenotypes for increased brown adipose tissue in mouse (salt stress response's intersection with sucrose stimulus response); and for human myopathy (red light response minus water deprivation response). We demonstrate the ranking of predictions for human holoprosencephaly from the set intersections between each pair of a variety of closely-related zebrafish phenotypes. Our results suggest that Boolean phenolog combinations may provide a more informed insight into the conserved pathways underlying diseases than either regular phenologs or the naïve Bayes approach.

Keywords - phenologs, deep homology, myopathy, breast cancer, methylmercury response, brown adipose tissue, autophagy, oxidative stress

\section{Background}

Limbs are an example of homologous structures - existing in multiple species - which appear to have evolved independently, but share underlying sets of conserved genes [1,2]. This concept, known as deep homology [1, explains the remarkable convergent evolution of eyes several dozen times [3]; the underlying genetic programs responsible for eye development must predate eyes 1]. It follows that such deeply conserved genetic processes played some selectively advantageous role in the most recent common ancestor and produced a measurable phenotype.

Phenologs are an extension of the homology of individual genes to sets of genes affiliated with specific structures, phenotypes, or diseases. McGary et al. defined phenologs as orthologous phenotypes - phenotypes from separate species which share an unexpectedly large number of associated genes (as determined by gene orthology). Furthermore, phenologs are mutually predictive; genes involved in one phenotype, but not known to play a role in a second, are predicted for the second, and vice versa [4. Woods et al. further demonstrated that gene-phenotype association predictions using phenologs can be improved by integrating information from multiple phenologs across several species [5]. If cases of structural homology have a non-structural common ancestor, phenologs fill in the theoretical gap for determining what that common ancestor might have been.

Using phenologs, we previously demonstrated the prediction and experimental validation of vertebrate neural crest development genes such as SEC23IP on the basis of the plant phenotype negative gravitropism defective [4]. Subsequently, we provided literature validation for genes we predicted for epilepsy and atrial fibrillation from a $k$ nearest neighbors search and Bayesian integration of orthologous phenotypes [5]. Whereas the $k$ nearest neighbors approach yielded a broad look at the orthologous processes which give rise to diseases and phenotypes, we present in this manuscript a more focused look. Both 
the phenolog and deep homology hypotheses are based on a modularity postulate: proteins and genes function together in pathways or functional modules which may be composed narrowly or broadly (and thus are divisible into more narrowly-defined functionalities). Notably, Bowers et al. identified triplets of proteins whose phylogenetic profiles - concurrent presence, absence, or combinations of the two - obey higher order relationships than have presently been explored using phenologs 6 . We were interested in determining whether these logical relationships hold true in phenotypic space as well as functional module space.

In this work, we describe the use of Boolean combinations of phenotypes to generate pseudo-phenotypes representing hypothetical functional modules, which are often more faithfully orthologous to the query diseases than were the original components. We demonstrate the prediction of genes associated with oxidative stress-related apoptosis in breast cancer from zebrafish, and increased brown adipose tissue as well as myopathy from plants. We also provide the source code and datasets.

\section{Methods}

\subsection{Matrix framework}

We utilized NMatrix, part of the SciRuby Project, for representation of sparse matrices in the Ruby language; the storage type is known as 'new' Yale 7 (described more thoroughly in 8 ), which stores only the diagonal and non-zero elements of each row.

As such, phenotypes may be represented as matrix rows and genes as columns; as in [5], cells containing 1 have an observed association between a gene and phenotype, and cells with 0 indicate no observation (as opposed to observed negative association, which is not included in our model). One gene-phenotype matrix is used to represent each species.

As in [5], INPARANOID orthogroups may be utilized to translate individual species-specific gene-phenotype matrices into sets of orthogroup-phenotype matrices, noting that each species pair requires a different gene-to-orthogroup translation. Rows with the same contents, which might be thought of as representing in-paralogous phenotypes, are collapsed together; consequently, all rows in a matrix are unique.

Within the resulting matrix, an all-versus-all search is performed, identifying all pairs of phenotypes which overlap. The selected operation (and or not) is carried out upon each overlapping pair. The latter operation is carried out twice, e.g., $A-B$ and $B-A$. If a resulting pseudo-phenotype is the same as either of the inputs, or consists of fewer than three orthogroups, it is discarded. Phenotypes with the exact same orthogroup sets are merged, as before.

Lastly, phenologs are identified between the target species and the computed matrix (as in [4]). A filter is imposed, throwing away all phenologs with an intersection size of less than two. In addition, phenologs which predict no new genes 


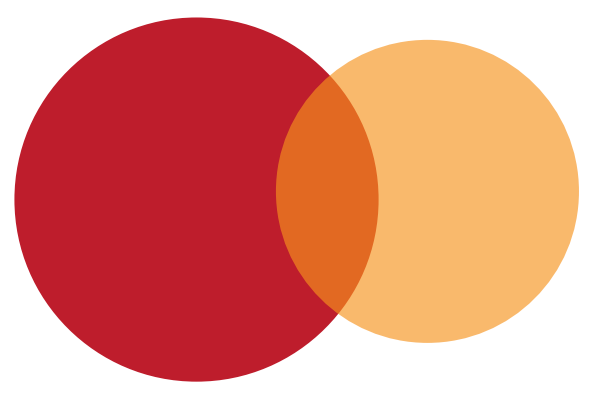

\section{Make boolean phenotypes by looking for overlapping phenotypes within a species}
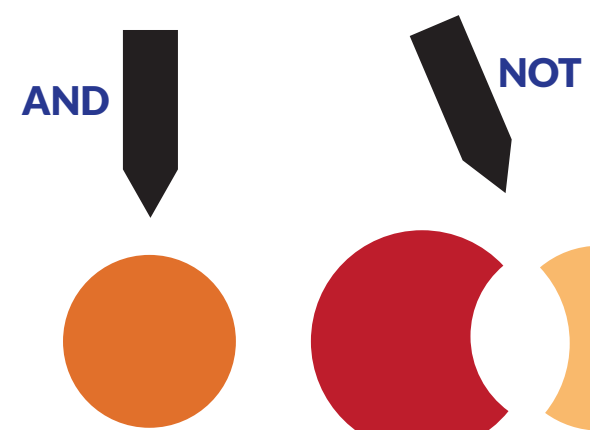

Find phenologs between new phenotypes and species-of-interest phenotypes

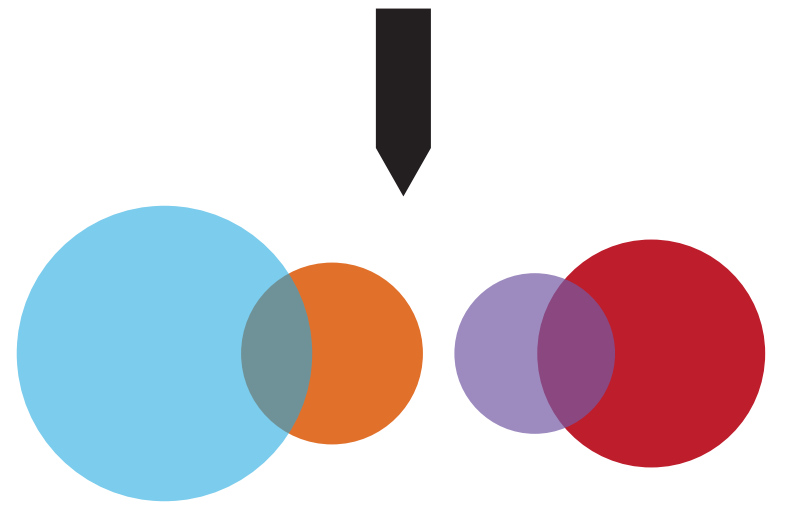

Figure 1: Process for the identification of Boolean phenologs. While Boolean combinations of phenotypes may be identified using any of the Boolean operators, we examine and and not combinations, as these are likely to provide relatively narrower views of the underlying conserved processes. 
(the intersection consists of the entire Boolean phenotype) are discarded. The orthogroups within the pseudo-phenotype but outside the overlap are translated into target-species gene identifiers and are considered to be candidates.

\subsection{Correction for multiple hypothesis testing}

To address the problem of multiple hypothesis testing, we performed an empirical permutation test similar to that used in [4]. We experimented with two different schemes and found that they produced similar results.

In both schemes, the psuedo-phenotype-orthogroup matrices from Figure 1 are used. In one, a single permutation is generated of an entire matrix and each row (phenotype) has its contents rearranged according to that same permutation. In the other scheme, each row is permuted separately.

In each scheme, phenologs are calculated for the matrix permutation; the process is repeated 1,000 times. The resulting distributions are plotted as in Figures 2 3, and a positive predictive value is calculated [9] for every phenolog as in [4].

Since the results were similar, we elected to use the single-permutation scheme, as it preserves the joint distribution of genes and phenotypes.

\section{Results}

\subsection{Hypothesis}

Diseases may consist of multiple phenotypes; such diseases are called syndromes. The definition of "phenotype" requires that they be observable [10, but we wondered if it might be helpful to separate phenotypes - as syndromes may be divided - into components. The components might be thought of as potentially arising from errors in distinct functional modules of genes. We hypothesized that it might be possible to identify such modules by performing various mathematical set operations (and, or, xor, not) on overlapping phenotype gene sets. Reasoning that or $(\cup$, union) and xor would produce broader rather than narrower views of phenotype orthology, we elected to consider and ( $\cap$, intersection) and not (-, subtraction) most carefully (Figure 1). In this paper, we present tests of the Boolean phenolog computational strategy and describe several examples in detail, accompanied by literature support for the predicted candidate genes where available.

\subsection{Increased brown adipose tissue from Arabidopsis}

To demonstrate the power of this method, we present the first highly ranked phenotype prediction from the first test run (mouse phenotypes from Arabidopsis combination phenotypes using the Boolean and operation). The mouse phenotype increased brown adipose tissue amount is well-predicted from the intersection of the plant GO biological processes response to salt stress $\cap$ response to sucrose stimulus (Figure 4). 


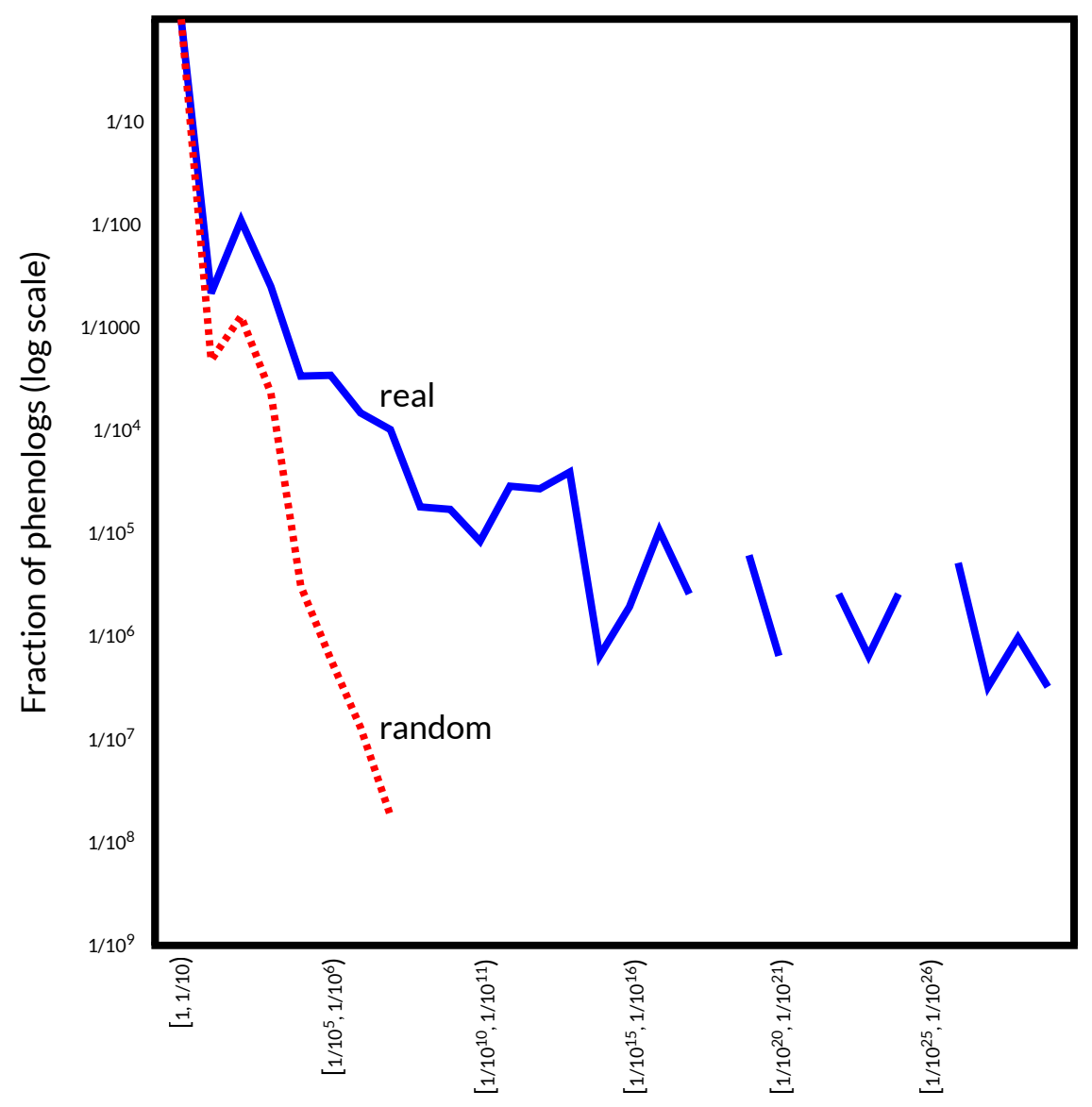

Hypergeometric frequency of observing overlap by chance

Figure 2: Real and null distributions based on a permutation test of HumanZebrafish Boolean not phenologs. The solid blue line represents the real distribution of $p$ values between phenotypes in a target species and Boolean phenotypes in a source species. The null distribution is based on 1,000 independent runs, permuting each matrix as a unit rather than each row independently. The distributions shown are for predicting human from zebrafish using the not operation. 

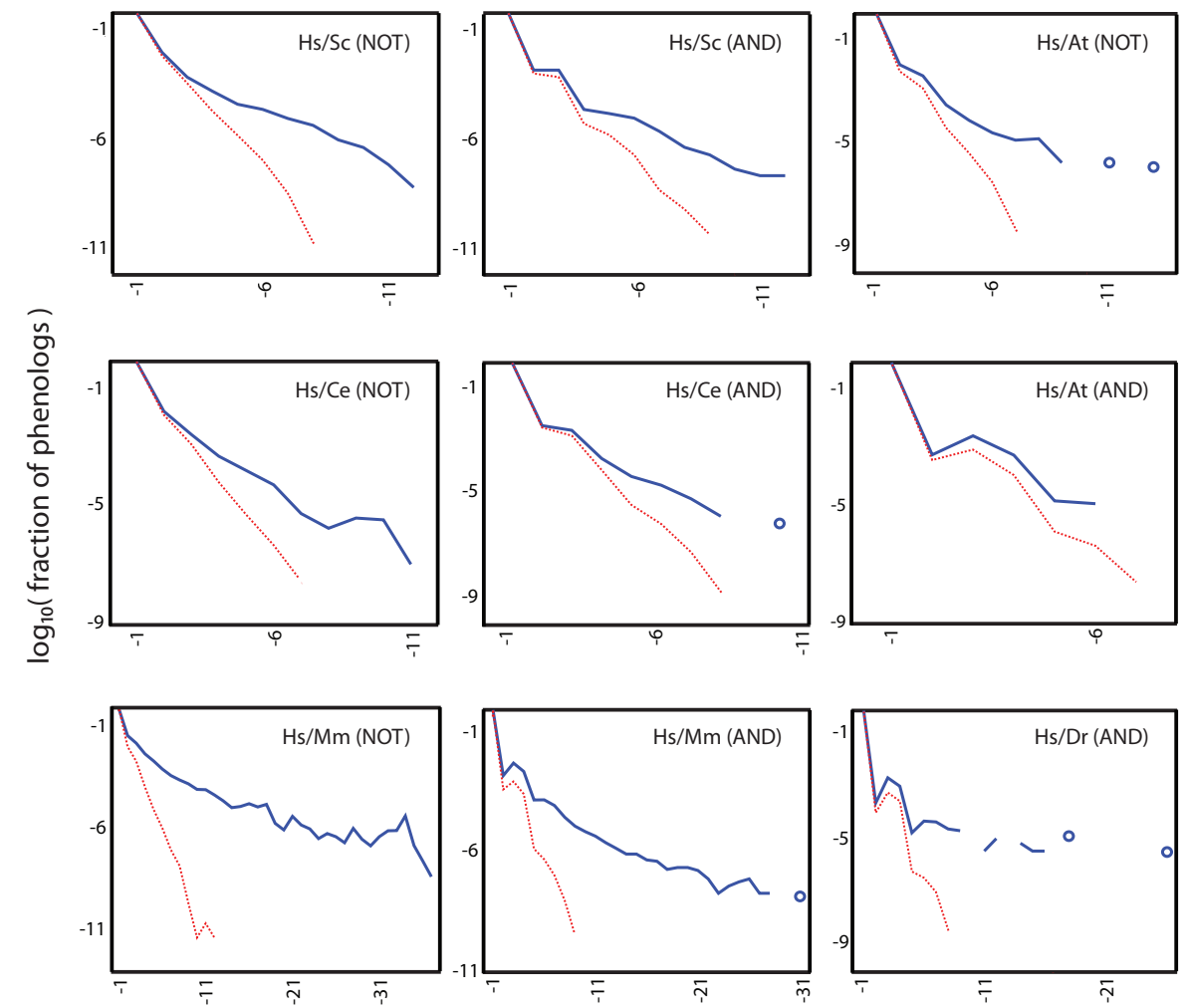

$\log _{10}$ ( Hypergeometric frequency of observing overlap by chance ) bin

Figure 3: Real and null distributions based on a permutation tests of Boolean phenologs. As with the human-zebrafish not permutation test, the real data offer more extreme hypergeometric frequencies than randomized data, with a generally greater separation between real and random on the not combinations than set intersection phenologs. Each $x$ axis bin spans a single $\log _{10}$ order of magnitude, with the labels corresponding to the more negative exponent in the bin boundary. The solid blue lines and circles represent the real distribution of $p$ values between phenotypes in a target species and Boolean phenotypes in a source species. The null distributions, the dashed red line, are based on 1,000 independent runs, permuting each matrix as a unit rather than each row independently. The distributions shown are for predicting human (Hs) from mouse $(\mathrm{Mm})$, yeast (Sc), A. thaliana (At), C. elegans (Ce), and zebrafish (Dr), using the not and and operations. 


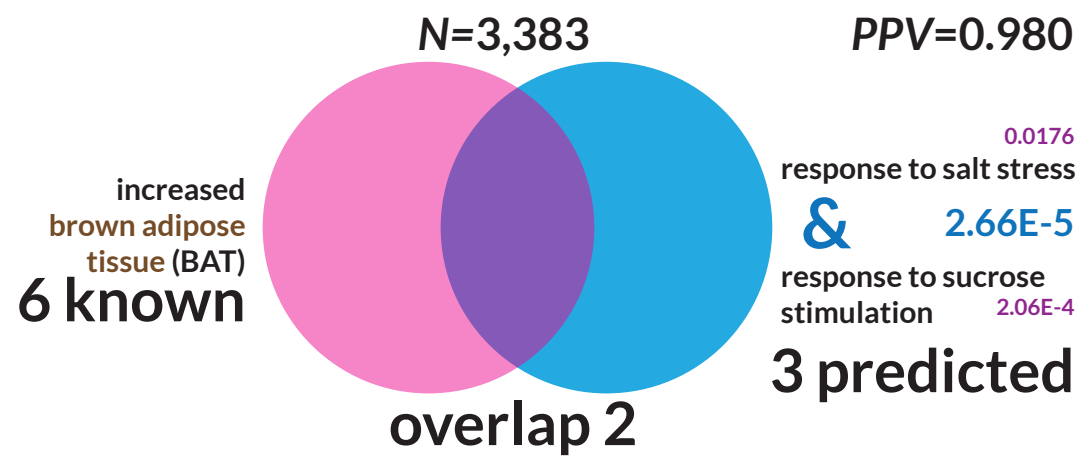

Figure 4: Mouse increased brown adipose tissue genes may be predicted by Arabidopsis Boolean phenotype response to salt stress $\cap$ response to sucrose stimulus. This figure represents the first mouse result outputted by the search of Arabidopsis phenotype intersections. The given intersection is the single nearest neighbor. The probability of seeing such an overlap (or larger) by chance is $p \leq 10^{-5}(\mathrm{FDR}=0.980)$, smaller by several orders of magnitude than the probability of seeing either of the individual phenologs separately $\left(p \leq 10^{-2}\right.$ for salt stress alone and $2 \times 10^{-4}$ for sucrose stimulus, neither of which meets our significance threshold of $\left.p<10^{-4}\right)$. The intersection size is two, with set sizes of six and five; thus, three orthogroups are predicted for the increased brown adipose tissue phenotype.

The most consequential difference between brown and white adipose tissue (BAT and WAT) is that the former dissipates energy as heat while the latter stores it. The uncoupling of mitochondrial oxidative phosphorylation in BAT is accomplished by uncoupling protein $U C P 1$, the molecular site of non-shivering thermogenesis [11. Drug-induced uncoupling has been pursued as a treatment for obesity, sometimes with lethal consequences 12,13 . A better understanding of BAT versus WAT physiology might potentially be useful for addressing the obesity epidemic.

The three genes predicted for increased BAT were Pgd (6-phosphogluconate dehydrogenase), Psmd4 (part of the 26S proteasome), and a large orthogroup of homeobox proteins (including Pitx1-3, Isx, Pax2-8, Rax, Alx3, Esx1, Crx, Otx1/2, Phox2a/b, and Sebox).

Phosphogluconate dehydrogenase is a lipogenic enzyme in the pentose phosphate pathway, and seems to be expressed in WAT, BAT, and liver, with activity varying according to sex and tissue [14]. Pankiewicz et al. also found that oestradiol regulates liver Pgd expression; whereas Puerta et al. found that oestradiol decreased BAT thermogenesis only in cold-acclimated rats 15 . While the full story would require a full literature review to elucidate, Pgd seems to be an interesting candidate.

The case for Psmd4 is only slightly clearer. UCP1 ubiquitinylation is associated with BAT in cold-acclimated animals, and ubiquitinylation seems to control the rate of UCP1 turnover by the proteasome 16. It is therefore possible that Psmd4 plays a direct role in BAT thermogenesis. 


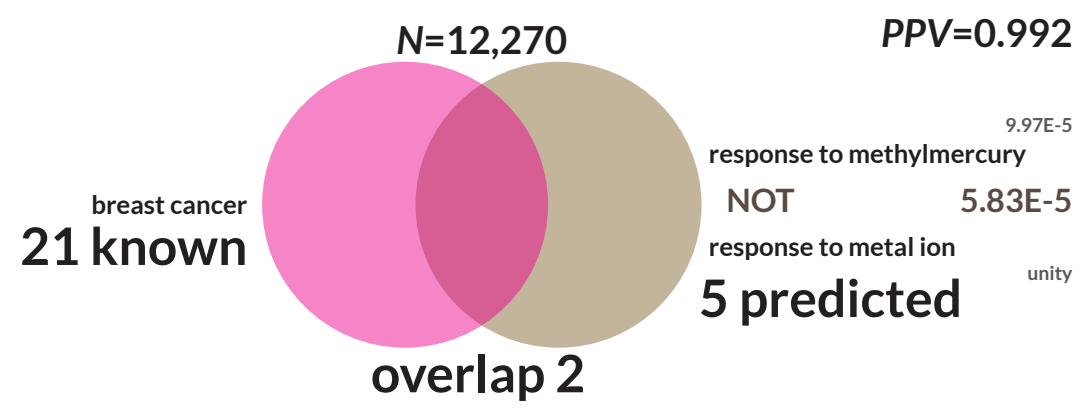

Figure 5: Breast cancer is predicted from genes involved in zebrafish methylmercury response but not response to metal ion. Five genes were predicted for breast cancer. The phenolog overlap was of size two, and nineteen known breast cancer genes were missed. The probability of seeing an overlap between breast cancer and defective methylmercury response alone is $p \leq 10^{-4}$.

Regarding predictions in the third group, homeobox genes are involved in adipogenesis, but it's unclear whether any of the candidates play a role; these too may be worthy of additional exploration.

In short, candidate genes can be predicted from plant combination phenotypes at high predictive values $(\mathrm{PPV}=0.980)$ and may be worthy of additional exploration.

\subsection{Set difference: methylmercury and breast cancer}

Next, we looked at Boolean combinations consisting of set differences, reasoning that these would identify more narrowly-defined modules rather than the larger assemblies of processes in which those specific modules play a role.

Perhaps the most striking example of the applicability of this technique, and of Boolean phenologs in general, is the case of human breast cancer being predicted from response to methylmercury less response to metal ion $(\mathrm{PPV}=$ $0.992, p \leq 10^{-5}$; Figure 5). We expected that the genes predicted would by DNA repair-related, as with many cancer phenologs (for example, breast cancer has a phenolog in plants with the intersection of DNA repair and response to gamma radiation, $p \leq 10^{-5}$, with two predicted orthogroups: ATR and ERCC6, which both appear to be DNA repair genes). Instead, these genes highlight two other pathways by which organisms (or individual cells) suppress cancer growth: apoptosis and oxidative stress response.

Methylmercury $\left(\mathrm{MeHg}^{+}\right.$or just $\mathrm{MeHg}$ ) is an organometallic cation, so this example appears at first glance to be a strange combination. However, the response to methylmercury GO annotation is a child node of response to organic substance in the directed acyclic graph, not response to metal ion. A recent article by McElwee et al. examines the effects of organic $(\mathrm{MeHgCl})$ and inorganic mercury $\left(\mathrm{HgCl}_{2}\right)$ on $C$. elegans, finding eighteen genes which were important to mercurial exposure response - and only two which responded to both types 
of mercury [17. The mechanisms for mercury toxicity are incompletely understood, but it seems clear that even if the two mechanisms are the same or similar (as argued by Clarkson et al. [18]), the organismal and cellular responses differ. Thus, we believe this phenolog to be consistent with the findings of McElwee et al. [17.

Five genes are predicted: $M T-C O I, J U N$ (c-Jun), SOD2, GADD45B, and $B A X$. All of these genes - MT-COI especially - appear to be involved in the apoptotic response to reactive oxygen species (ROS). The mechanisms by which methylmercury generates ROS are not entirely clear, as previously mentioned, but are reviewed by Farina et al. 19 . Generally, $\mathrm{MeHg}^{+}$forms a complex with lower-weight thiol and selenol groups, especially glutathione (reviewed separately in $[20 \mid$ ). When such groups occur in mitochondrial creatine kinase or respiratory chain proteins, the compound can inhibit mitochondrial function, leading to depolarization of that organelle's membrane and overproduction of ROS [19].

In the case of a malfunction of any one of the predicted genes, the effects of ROS may be magnified. Mitochondria rely on SOD2 (Mn-SOD; reviewed in [21]) for conversion of extremely toxic superoxide into molecular oxygen and hydrogen peroxide, which can be eliminated by catalase. Several of the genes ( $J U N$, reviewed in 22 ; and $G A D D 45 B$ and $B A X$, reviewed in relation to p53 in [23]) play well-characterized roles in stress-induced apoptosis, triggered in the event the cell is overwhelmed by free radicals (or other agents which cause damage).

MT-COI, part of complex IV of the oxidative phosphorylation pathway, is the site of an extremely common germ line mutation in cancer patients, which seems to predispose those individuals toward developing cancer [24. The occurrence of this mutation may suggest a role for this cytochrome $\mathrm{C}$ oxidase component in the pro-apoptotic pathway.

Induction of apoptosis is a key route by which chemotherapy targets cancers - and by which cancers circumvent chemotherapy. The roles of Bcl-2 and $\mathrm{Bax}$ are reviewed in 25]. Notably, Bcl-2 binds Bax (the protein products of $B C L$-2 and our prediction $B A X$, respectively), and $B C L$ - 2 over-expression confers chemotherapy resistance. When Bcl-2 is low or absent, however, Bax homodimerizes, leading to cell death. Bax is also thought to interact with the mitochondrial voltage-dependent ion channel, and is directly induced by p53 in response to DNA damage.

Interestingly, a model already exists that might explain these breast cancer gene predictions. Martinez-Outschoorn et al. proposed, in 2010, the "autophagic tumor stroma model of cancer." Essentially, the model hypothesized that tumors induce oxidative stress in the tumor microenvironment in order to cause stromal cells to release nutrients which are used for cancer growth 26, 27]. Indeed, Trimmer et al. found that loss of Caveolin-1 (Cav-1) — whose stromal presence is a strong predictor of survival - dramatically promotes breast cancer growth. Loss of Cav-1 may be rescued by over-expression of SOD2, another tumor suppressor, which relieves oxidative stress by processing mitochondrial superoxide radicals. 27 


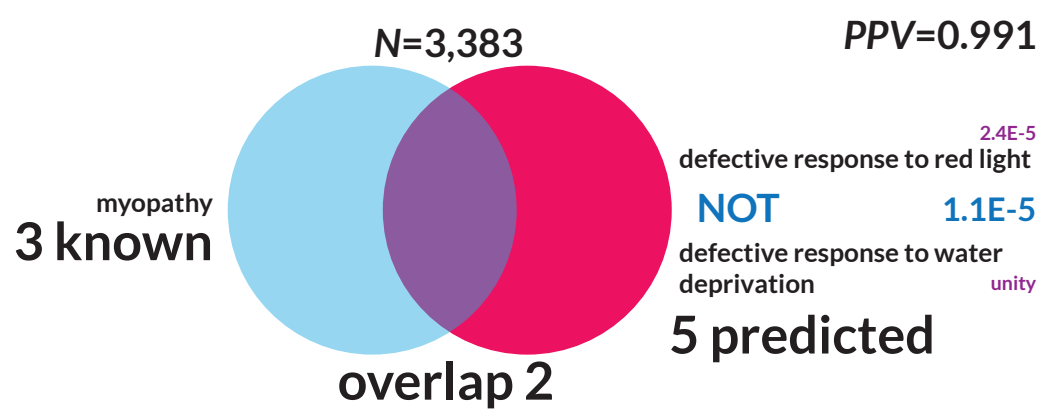

Figure 6: Myopathy is predicted from plant genes involved in red light response but not response to water deprivation. Among 3,383 human-plant orthogroups, three are involved in myopathy; five in the Boolean phenolog defective response to red light less defective response to water deprivation; and two in the intersection $\left(p \leq 10^{-5}, P P V=0.987\right)$. The sub-phenolog myopathy / red light has $p \leq 10^{-5}$.

$G A D D 45 B$ also plays a pro-apoptotic role, and was found to be downregulated in at least two cases of hepatocellular carcinomas (reviewed in [28]). Both $G A D D 45 B$ and to a lesser extent SOD2 were observed to be up-regulated in inflammatory breast cancer (IBC) $[29$. The differential regulation argues for a $G A D D 45 B$ cancer role, whether as an oncogene or a tumor suppressor; and evidence in mice suggests that all of the Gadd45 genes, including Gadd45b, are involved in cancer immune response and are tumor suppressors [30].

These observations suggest further investigations into MT-COI's role in apoptosis and oxidative stress response - and hint that all of the predicted genes may be involved in a tumor suppression transcriptional program.

\subsection{Myopathy from Arabidopsis}

Myopathy is a broad category of disease categorized by muscular weakness with twenty-one associated human genes; three of these genes (DYSF, ACTA1, and FHL1) are members of human-plant orthogroups.

Using the Boolean analysis, we find that the combined Arabidopsis phenotype response to red light less response to water deprivation, predicts the involvement of five orthogroups in myopathy $\left(\mathrm{PPV}=0.991 ; p \leq 10^{-5}\right.$; Figure 6). The first of these orthogroups is comprised of members of the SWI/SNF complex and Mediator, including MED25, ARID1A (BAF250A), ARID1B (BAF250B), and PTOV1. MED25 has been observed in a family with Charcot-Marie-Tooth (CMT) syndrome, including childhood onset distal muscle weakness 31.

$A R I D 1 A$ and $A R I D 1 B$ are both muscle-related. In knockouts of the former, relatively fewer skeletal muscle cells differentiate from embryonic stem cells compared to other differentiation products $[32$. ARID1B is associated with CoffinSiris syndrome, which includes hypotonia among its symptoms 33 .

In the second orthogroup, two of the three genes are involved in mus- 
cle. CREM is expressed in ventricular myocytes 34 and $A T F 1$ is a hypoxiaresponsive transcriptional activator of skeletal muscle via mitochondrial UCP3 [35]; the third, CREB1, is expressed in fibroblasts and not in myocytes 34].

The third set of predictions is TFEB, TFEC, TFE3, and MITF. TFEC is a transcriptional activator of non-muscle myosin, and so is ruled out - but is nevertheless an interesting prediction. Over-expression of TFEB relieves Pompe disease, a disability of heart and skeletal muscles, by stimulating autophagy [36]. MITF has a known autophagy role resembling that of TFEB [37] and is involved in a variety of developmental processes, but no evidence exists suggesting it or TFE3 are involved in myopathy.

An autophagy link for these predictions is further supported by the fourth predicted orthogroup, consisting of a number of cytochrome P450 (family 2) genes: CYP2A6-17/13, CYP2B6, CYP2C8-9/18-19, and CYP2D6/E1/F1/ $J 2 / S 1 / U 1 / R 1 / W 1$. Cytochrome P450 is the site of a number of drug interactions - notably with grapefruit, cranberry, and pomegranate juice, which inhibit CYP3A4, a metabolizer of statins. CYP2C8, CYP2C9, and CYP2C19 are involved in various statin-induced myopathies 38 40. At least with the last of these, the mechanism is autophagy related [41. Similarly, predicted gene $C Y P 2 D 6$ increased statin efficacy and is a predicted drug interaction site with $3 A 4$ [42]. Finally, CYP2E1 metabolizes ethanol - which also causes myopathy - and inhibits autophagy $43[45]$.

Two genes for which we found no literature support are FAM50A and FAM50B, predicted as a single orthogroup; neither appears to be particularly well researched. These may be good candidates for autophagy genes.

There are at least two other Boolean phenologs of myopathy (both intersectional rather than subtractive) at $p \leq 10^{-6}(P P V=0.987)$. The first of these is response to light stimulus $\cap$ response to red light $\left(p \leq 10^{-4}\right.$ and $10^{-5}$ for the individual components), which predicts the cytochrome P450 orthogroup. The second is response to auxin stimulus $\cap$ response to light stimulus (the former component has $p \leq 10^{-4}$ ), predicting $G H D C$. We found no literature support for $G H D C$, a gene about which little is known; it may thus be an interesting candidate for myopathy and autophagy.

\subsection{Holoprosencephaly}

Finally, in order to demonstrate predictions from multiple phenologs and to show the utility of intersection phenologs, we present an example of a disease with many phenologs among the and (intersection) pseudo-phenotypes.

The concept of $k$ nearest neighbors-based ranking discussed in previous work [5] is analogous to the $k=1$ case of Boolean or (set union) phenologs. We present here an example of and (set intersection) phenologs for $k=1$, with candidate genes for holoprosencephaly (HPE) predicted from $D$. rerio intersection phenologs (see Table 1). In this case, many phenologs are supported by similarly good $p$ values, and predict a variety of genes (see Table 2 for $k$ NN-based rankings). It is worth noting that no single component's $p$ value is better than the Boolean $p$ value. 


\begin{tabular}{|c|c|c|}
\hline phenotype $A$ & phenotype $B$ & candidates \\
\hline brain development & $\begin{array}{c}\text { axon guidance } \\
\text { muscle organ development }\end{array}$ & $\begin{array}{l}\text { LHX2 } \\
\text { PRKCI }\end{array}$ \\
\hline dorsal/ventral pattern formation & $\begin{array}{l}\text { floor plate formation } \\
\text { adenohypophysis development }\end{array}$ & $\begin{array}{l}\text { NODAL } \\
\text { FGF3 }\end{array}$ \\
\hline somitogenesis & $\begin{array}{l}\text { nervous system development } \\
\text { endocrine pancreas development }\end{array}$ & $\begin{array}{l}\text { NEO1 } \\
W N T 5 A / B\end{array}$ \\
\hline muscle organ development & $\begin{array}{l}\text { muscle cell fate specification } \\
\text { blood circulation } \\
\text { somitogenesis }\end{array}$ & \multirow[t]{2}{*}{$S C U B E 1 / 3$} \\
\hline somitogenesis & $\begin{array}{l}\text { blood circulation } \\
\text { muscle cell fate specification }\end{array}$ & \\
\hline adenohypophysis development & $\begin{array}{c}\text { blood circulation } \\
\text { muscle cell fate specification }\end{array}$ & $D I S P 1$ \\
\hline blood circulation & $\begin{array}{c}\text { axon guidance } \\
\text { striated muscle cell development }\end{array}$ & CXCL12 \\
\hline axon guidance & $\begin{array}{c}\text { nervous system development } \\
\text { floor plate formation } \\
\text { muscle organ development } \\
\text { endocrine pancreas development } \\
\text { embryonic digestive tract morphogenesis }\end{array}$ & \multirow{7}{*}{$S M O$} \\
\hline nervous system development & $\begin{array}{c}\text { floor plate formation } \\
\text { muscle organ development } \\
\text { endocrine pancreas development } \\
\text { embryonic digestive tract morphogenesis }\end{array}$ & \\
\hline floor plate formation & $\begin{array}{c}\text { muscle organ development } \\
\text { adenohypophysis development } \\
\text { embryonic digestive tract morphogenesis }\end{array}$ & \\
\hline muscle organ development & $\begin{array}{l}\text { endocrine pancreas development } \\
\text { adenohypophysis development } \\
\text { embryonic digestive tract morphogenesis }\end{array}$ & \\
\hline endocrine pancreas development & $\begin{array}{l}\text { adenohypophysis development } \\
\text { embryonic digestive tract morphogenesis }\end{array}$ & \\
\hline adenohypophysis development & embryonic digestive tract morphogenesis & \\
\hline diencephalon development & neural plate morphogenesis & \\
\hline
\end{tabular}

Table 1: Holoprosencephaly genes are predicted by many intersection phenologs at the same $p$ value. We include holoprosencephaly as an example because it has many Boolean and phenologs from zebrafish at the same $p$ value $\left(6 \times 10^{-7}\right.$, which is the lowest $p$ value meeting the filtering criteria). Phenotypes $A$ and $B$ are arbitrarily labeled and indistinguishable overall (but not within individual pairs of table rules). Consider the first entry: brain development and axon guidance predicts gene LHX2; but brain development and muscle organ development predicts PRKCI. Nineteen different Boolean phenologs predict Smoothened. Rows are grouped first by candidate genes, but also by phenotype $A$ when candidate genes differ. PPV $=0.999$. 


\begin{tabular}{cc}
\hline orthogroup members & magnitude \\
\hline$S M O$ & 19 \\
\hline$S C U B E 1 / 3$ & 5 \\
\hline$D I S P 1$ & 2 \\
$C X C L 12$ & 2 \\
\hline$N E O 1$ & 1 \\
$W N T 5 A / B$ & 1 \\
$F G F 3$ & 1 \\
$N O D A L$ & 1 \\
$L H X 2$ & 1 \\
$P R K C I$ & 1 \\
\hline
\end{tabular}

Table 2: Holoprosencephaly candidate genes may be ranked by evidence. We ranked by hand the genes suggested for testing from the phenotypes in Table 1 as they would be ranked by either the naïve Bayes or additive classifiers in the previous Chapter. We included the magnitude (the number of times the gene is predicted by a phenolog in Table 11). Genes within ranks are predicted with the same score (e.g., NEO1 and LHX2) and are thus equally likely under this model). The best predicted gene is $S M O$, also known as Smoothened.

The most highly predicted gene is SMO (Smoothened), which is indeed an HPE gene - as determined by Rosenfeld et al. in 2010 [46], subsequent to the creation of our database. Rosenfeld et al. also identified DISP1, ranked third. NODAL, one of the bottom-ranked genes, has been observed as promoting an HPE-like phenotype in chick embryos [47] and mice (reviewed in [48]). Another gene ranked with NODAL, LHX2 ( $\operatorname{Lim} 1 / L h x 2)$, is required for mouse head formation [49] and seems to regulate the development of the midline of the brain in that species 50 .

The second-ranked orthogroup, consisting of SCUBE1 and SCUBE3, is suggested for HPE candidacy by its role in mouse brain formation (specifically SCUBE1) 51, but does not appear to be directly associated with HPE.

\subsection{Discussion}

Boolean phenologs offer a marked improvement over the $k$ nearest neighbors approach described previously 5 . The basis for the improvement is not entirely clear, but may be partially subjective: we selected preferentially those Boolean phenologs where the $p$ value is less than both the components. Nonetheless, for the not phenologs, all Boolean $p$ values are more significant at our maximum $p$ threshold of $1 \times 10^{-4}$. This property is made more likely, but not guaranteed, by the mathematics of set intersection probabilities. If the subtracted phenotype intersects with the query, the Boolean phenotype will have a smaller intersection as a result of the subtraction; and thus the $p$ value is more likely to rise above 
our threshold. The $p$ value of two sets with no intersection is within $\epsilon$ of unity.

A minor contributor is the way in which the genes in the single-species matrices are translated into orthogroups. For example, plant phenotypes regulation of telomere maintenance and regulation of chromosome organization have differing sets of genes; but when the genes are translated into human-plant orthogroups, and those plant genes without human orthologs are removed, the two phenotypes collapse into one (these are in-paralogs in $A$. thaliana with respect to H. sapiens).

The filtering procedure also plays a role. The original phenolog method looked for any intersection at all; we require an intersection of size two or larger, and additionally that at least one new orthogroup is predicted. For and phenotypes, these last two criteria imply that the intersection between the Boolean components must be at least size three.

The probability of finding such a three-way intersection by chance is quite low, and would ordinarily be described by the multivariate hypergeometric distribution (thus, the given probabilities may be conservative over-estimates). The frequency of the three-way intersections - higher than would be expected at random - is a product of the way in which gene-phenotype associations are discovered by biologists.

It follows, then, that the standard hypergeometric distribution may not be conservative enough for phenologs that are "circular". We define circular by way of an example. Bardet-Biedl syndrome candidate genes which were identified subsequent to the assembly of our gene-phenotype association database are perfectly predicted by the zebrafish Boolean phenotype melanosome transport less embryonic specification defect $\left(p \leq 10^{-25}\right)$. However, zebrafish melanosome transport is studied at least in part for its role in Bardet-Biedl syndrome. As such, melanosome transport-associated genes at the time of database construction were already only one patient validation away from being Bardet-Biedlassociated genes. Circularity may explain the unusually extreme hypergeometric $p$ values in the human-mouse Boolean phenologs (see Figure 3).

An additional concern is over-training. One might argue, for example, that by subtracting every phenotype from every other phenotype, and then looking for phenologs, one is simply trimming away some of the less immediately relevant portions of phenotype gene sets; however, Boolean phenologs simply provide good hints about which genes and processes should be studied for a deep homology role. Some genes may be missed, but the advantage is an observably lower false positive rate. As with any predictive methodology, we view Boolean phenologs as hypothesis generators, suggesting starting points for deeper investigation.

A final issue is confirmation bias, particularly when pursuing literature validation. Ioannidis argued in 2005 that most published research findings are false positives, in part due to the failure of researchers to share negative results (which are more likely to be correct) [52. Indeed, our gene-phenotype association matrices only contain "positive" and "unobserved," and lack negatives. Negatives would be extremely useful for evaluation of results as well. Particularly when predicting extremely well-studied diseases like cancer, it's unlikely 
that one will find a well-characterized gene which is not in some way associated if one looks long enough. One potential approach might be is to silently insert an additional random prediction in some set fraction of phenologs selected for literature validation, and then determine how frequently the random prediction is marked as true by the researcher.

\section{Conclusions}

Boolean phenologs offer a computational approach to calculate phenologs [4,5], in a manner designed to focus attention on specific component modules and subprocesses which underlie diseases and phenotypes, as well as the non-obvious homologies which exist between organisms. Here, we have presented Boolean phenolog models for human diseases such as myopathy and breast cancer, as well as increased brown adipose tissue in mice. We describe a number of predictions worthy of further testing, including FAM50A and FAM50B for autophagy and $M T$-COI for oxidative stress and apoptosis in cancer.

Notably, as with phenologs, the Boolean approach offers insight into those elements of diseases, traits, or processes which are conserved and well-studied. A human-zebrafish phenolog is informative about breast cancer only insofar as cancer is affected by well-characterized processes shared between the two organisms. Phenologs are incapable of highlighting the uniquely human components of breast cancer, but can give us information about the roles of oxidative stress and apoptosis — or about DNA repair genes — in cancer.

\section{Availability of source code and data}

Gene-phenotype associations are available at the phenologs website, phenologs . org. The source code and documentation are available on GitHub at http: //github.com/marcottelab/boolean.

\section{Competing interests}

The authors declare that they have no competing interests.

\section{Authors' contributions}

J.O.W. performed the analyses and drafted the manuscript. M.T. performed preliminary analyses. E.M.M. supervised the research. All authors edited the final manuscript. 


\section{Acknowledgements}

This work was supported by grants from the Cancer Prevention Research Institute of Texas, the National Science Foundation, the National Institutes of Health, and the Welch Foundation (F-1515); and a National Science Foundation Graduate Research Fellowship (to J.O.W.).

\section{References}

[1] Shubin, N., Tabin, C., Carroll, S.: Fossils, genes and the evolution of animal limbs. Nature 388(6643), 639-48 (1997). doi:10.1038/41710

[2] Shubin, N., Tabin, C., Carroll, S.: Deep homology and the origins of evolutionary novelty. Nature 457(7231), 818-23 (2009). doi:10.1038/nature07891

[3] von Salvini-Plawen, L., Mayr, E.: On the evolution of photoreceptors and eyes. Evolutionary Biology 10 (1977)

[4] McGary, K.L., Park, T.J., Woods, J.O., Cha, H.J., Wallingford, J.B., Marcotte, E.M.: Systematic discovery of nonobvious human disease models through orthologous phenotypes. Proceedings of the National Academy of Sciences of the United States of America 107(14), 6544-9 (2010). doi:10.1073/pnas.0910200107

[5] Woods, J.O., Singh-Blom, U.M., Laurent, J.M., McGary, K.L., Marcotte, E.M.: Prediction of gene-phenotype associations in humans, mice, and plants using phenologs. BMC bioinformatics 14(203), 1-17 (2013). doi:10.1186/1471-2105-14-203

[6] Bowers, P.M., Cokus, S.J., Eisenberg, D., Yeates, T.O.: Use of logic relationships to decipher protein network organization. Science 306, 2246-9 (2004). doi:10.1126/science. 1103330

[7] Eisenstat, S.C., Elman, H.C., Schultz, M.H., Sherman, A.H.: The (New) Yale Sparse Matrix Package. In: Birkhoff, G., Schoenstadt, A.L. (eds.) Elliptic Problem Solvers II, pp. 45-52. Academic Press, Inc. (1984)

[8] Bank, R.E., Douglas, C.C.: Sparse matrix multiplication package (SMMP). Advances in computational mathematics 1(1), 127-137 (1993)

[9] Benjamini, Y., Hochberg, Y.: Controlling the false discovery rate: a practical and powerful approach to multiple testing. Journal of the Royal Statistical Society: series B (methodological) 57(1), 289-300 (1995)

[10] Johannsen, W.: The genotype conception of heredity. The American naturalist 45(531), 129-159 (1911) 
[11] Nicholls, D.G., Bernson, V.S., Heaton, G.M.: The identification of the component in the inner membrane of brown adipose tissue mitochondria responsible for regulating energy dissipation. Experientia. Supplementum 32, 89-93 (1978)

[12] McFee, R.B., Caraccio, T.R., McGuigan, M.A., Reynolds, S.A., Bellanger, P.: Dying to be thin: a dinitrophenol related fatality. Veterinary and human toxicology 46(5), 251-4 (2004)

[13] Miranda, E.J., McIntyre, I.M., Parker, D.R., Gary, R.D., Logan, B.K.: Two deaths attributed to the use of 2,4-dinitrophenol. Journal of analytical toxicology 30(3), 219-22 (2006)

[14] Pankiewicz, A., Sledzinski, T., Nogalska, A., Swierczynski, J.: Tissue specific, sex and age-related differences in the 6-phosphogluconate dehydrogenase gene expression. The International Journal of Biochemistry \& Cell Biology 35(2), 235-45 (2003)

[15] Puerta, M.L., Nava, M.P., Abelenda, M., Fernández, A.: Inactivation of brown adipose tissue thermogenesis by oestradiol treatment in coldacclimated rats. Pflügers Archiv : European journal of physiology 416(6), 659-62 (1990)

[16] Clarke, K.J., Adams, A.E., Manzke, L.H., Pearson, T.W., Borchers, C.H., Porter, R.K.: A role for ubiquitinylation and the cytosolic proteasome in turnover of mitochondrial uncoupling protein 1 (UCP1). Biochimica et biophysica acta 1817(10), 1759-67 (2012). doi:10.1016/j.bbabio.2012.03.035

[17] McElwee, M.K., Ho, L.A., Chou, J.W., Smith, M.V., Freedman, J.H.: Comparative toxicogenomic responses of mercuric and methyl-mercury. BMC genomics 14(1), 698 (2013). doi:10.1186/1471-2164-14-698

[18] Clarkson, T.W., Magos, L.: The toxicology of mercury and its chemical compounds. Critical reviews in toxicology 36(8), 609-62 (2006). doi:10.1080/10408440600845619

[19] Farina, M., Aschner, M., Rocha, J.a.B.T.: Oxidative stress in MeHginduced neurotoxicity. Toxicology and applied pharmacology 256(3), 40517 (2011). doi:10.1016/j.taap.2011.05.001

[20] Farina, M., Rocha, J.a.B.T., Aschner, M.: Oxidative Stress and Methylmercury-induced Neurotoxicity. In: Wang, C., Slikker, W. (eds.) Developmental Neurotoxicology Research: Principles, Models, Techniques, Strategies, and Mechanisms, pp. 357-385. John Wiley \& Sons, Hoboken, New Jersey (2011). Chap. 18.

[21] Zelko, I., Mariani, T., Folz, R.: Superoxide dismutase multigene family: a comparison of the CuZn-SOD (SOD1), Mn-SOD (SOD2), and EC-SOD (SOD3) gene structures, evolution, and expression. Free Radical Biology and Medicine 33(3), 337-349 (2002) 
[22] Chen, Y.R., Tan, T.H.: The c-Jun N-terminal kinase pathway and apoptotic signaling (review). International Journal of Oncology (2000). doi:10.3892/ijo.16.4.651

[23] Haupt, S., Berger, M., Goldberg, Z., Haupt, Y.: Apoptosis - the p53 network. Journal of cell science 116(Pt 20), 4077-85 (2003). doi:10.1242/jcs.00739

[24] Gallardo, M.E., Moreno-Loshuertos, R., López, C., Casqueiro, M., Silva, J., Bonilla, F., Rodríguez de Córdoba, S., Enríquez, J.A.: m.6267G¿A: a recurrent mutation in the human mitochondrial DNA that reduces cytochrome c oxidase activity and is associated with tumors. Human mutation 27(6), 575-82 (2006). doi:10.1002/humu.20338

[25] Reed, J.C., Miyashita, T., Takayama, S., Wang, H.G., Sato, T., Krajewski, S., Aimé-Sempé, C., Bodrug, S., Kitada, S., Hanada, M.: BCL-2 family proteins: regulators of cell death involved in the pathogenesis of cancer and resistance to therapy. Journal of cellular biochemistry 60(1), 23-32 (1996).

[26] Martinez-Outschoorn, U.E., Whitaker-Menezes, D., Pavlides, S., Chiavarina, B., Bonuccelli, G., Casey, T., Tsirigos, A., Migneco, G., Witkiewicz, A., Balliet, R., Mercier, I., Wang, C., Flomenberg, N., Howell, A., Lin, Z., Caro, J., Pestell, R.G., Sotgia, F., Lisanti, M.P.: The autophagic tumor stroma model of cancer or "battery-operated tumor growth:" A simple solution to the autophagy paradox. Cell cycle 9(21), 4297-306 (2010). doi:10.4161/cc.9.21.13817

[27] Trimmer, C., Sotgia, F., Whitaker-Menezes, D., Balliet, R.M., Eaton, G., Martinez-Outschoorn, U.E., Pavlides, S., Howell, A., Iozzo, R.V., Pestell, R.G., Scherer, P.E., Capozza, F., Lisanti, M.P.: Caveolin-1 and mitochondrial SOD2 (MnSOD) function as tumor suppressors in the stromal microenvironment: A new genetically tractable model for human cancer associated fibroblasts. Cancer biology \& therapy 11(4), 383-394 (2011). doi:10.4161/cbt.11.4.14101

[28] Salvador, J.M., Brown-Clay, J.D., Fornace, A.J.: Gadd45 in stress signaling, cell cycle control, and apoptosis. Advances in experimental medicine and biology 793, 1-19 (2013). doi:10.1007/978-1-4614-8289-5_1

[29] Lerebours, F., Vacher, S., Andrieu, C., Espie, M., Marty, M., Lidereau, R., Bieche, I.: NF-kappa B genes have a major role in inflammatory breast cancer. BMC cancer 8, 41 (2008). doi:10.1186/1471-2407-8-41

[30] Ju, S., Zhu, Y., Liu, L., Dai, S., Li, C., Chen, E., He, Y., Zhang, X., Lu, B.: Gadd45b and Gadd45g are important for anti-tumor immune responses. European journal of immunology 39(11), 3010-8 (2009). doi:10.1002/eji.200839154 
[31] Leal, A., Huehne, K., Bauer, F., Ekici, A., Pasutto, F., Endele, S.: Identification of the variant Ala335Val of MED25 as responsible for CMT2B2: molecular data, functional studies of the SH3 recognition motif and correlation between wild-type MED25 and PMP22 RNA levels in CMT1A animal models. Neurogenetics 10, 275-287 (2009). doi:10.1007/s10048-009-0183-3

[32] Gao, X., Tate, P., Hu, P., Tjian, R., Skarnes, W.C., Wang, Z.: ES cell pluripotency and germ-layer formation require the SWI/SNF chromatin remodeling component BAF250a. Proceedings of the National Academy of Sciences of the United States of America 105(18), 6656-61 (2008). doi:10.1073/pnas. 0801802105

[33] Santen, G.W.E., Aten, E., Vulto-van Silfhout, A.T., Pottinger, C., van Bon, B.W.M., van Minderhout, I.J.H.M., Snowdowne, R., van der Lans, C.a.C., Boogaard, M., Linssen, M.M.L., Vijfhuizen, L., van der Wielen, M.J.R., Vollebregt, M.J.E., Breuning, M.H., Kriek, M., van Haeringen, A., den Dunnen, J.T., Hoischen, A., Clayton-Smith, J., de Vries, B.B.A., Hennekam, R.C.M., van Belzen, M.J., Almureikhi, M., Baban, A., Barbosa, M.-f., Ben-Omran, T., Berry, K., Bigoni, S., Boute, O., Brueton, L., van der Burgt, I., Canham, N., Chandler, K.E., Chrzanowska, K., Collins, A.-n.L., de Toni, T.-e., Dean, J., den Hollander, N.-l.S., Flore, L.A., Fryer, A., Gardham, A., Graham, J.M., Harrison, V., Horn, D., Jongmans, M.C., Josifova, D., Kant, S.G., Kapoor, S., Kingston, H., Kini, U., Kleefstra, T., Krajewska-Walasek, M., Kramer, N., Maas, S.M., Maciel, P., Mancini, G.M.S., Maystadt, I., McKee, S., Milunsky, J.M., Nampoothiri, S., Newbury-Ecob, R., Nikkel, S.M., Parker, M.J., Pérez-Jurado, L.a., Robertson, S.P., Rooryck, C., Shears, D., Silengo, M., Singh, A., Smigiel, R., Soares, G., Splitt, M., Stewart, H., Sweeney, E., Tassabehji, M., Tuysuz, B., van Eerde, A.M., Vincent-Delorme, C., Wilson, L.C., Yesil, G.: Coffin-Siris Syndrome and the BAF Complex: Genotype-Phenotype Study in 63 Patients. Human mutation (2013). doi:10.1002/humu.22394

[34] Husse, B., Isenberg, G.: CREB expression in cardiac fibroblasts and CREM expression in ventricular myocytes. Biochemical and biophysical research communications 334(4), 1260-5 (2005). doi:10.1016/j.bbrc.2005.06.206

[35] Lu, Z., Sack, M.N.: ATF-1 is a hypoxia-responsive transcriptional activator of skeletal muscle mitochondrial-uncoupling protein 3 . The Journal of biological chemistry 283(34), 23410-8 (2008). doi:10.1074/jbc.M801236200

[36] Spampanato, C., Feeney, E., Li, L., Cardone, M., Lim, J.-A., Annunziata, F., Zare, H., Polishchuk, R., Puertollano, R., Parenti, G., Ballabio, A., Raben, N.: Transcription factor EB $(T F E B)$ is a new therapeutic target for Pompe disease. EMBO molecular medicine 5(5), 691-706 (2013). doi:10.1002/emmm.201202176

[37] Martina, J.A., Puertollano, R.: RRAG GTPases link nutrient availability to gene expression, autophagy and lysosomal biogenesis. Autophagy 9(6), 928-30 (2013). doi:10.4161/auto.24371 
[38] Gallelli, L., Ferraro, M., Spagnuolo, V., Rende, P., Mauro, G.F., De Sarro, G.: Rosuvastatin-induced rhabdomyolysis probably via CYP2C9 saturation. Drug metabolism and drug interactions 24(1), 83-7 (2009)

[39] Ishikawa, C., Ozaki, H., Nakajima, T., Ishii, T., Kanai, S., Anjo, S., Shirai, K., Inoue, I.: A frameshift variant of $C Y P 2 C 8$ was identified in a patient who suffered from rhabdomyolysis after administration of cerivastatin. Journal of human genetics 49(10), 582-5 (2004). doi:10.1007/s10038-004$0188-6$

[40] Marusic, S., Lisicic, A., Horvatic, I., Bacic-Vrca, V., Bozina, N.: Atorvastatin-related rhabdomyolysis and acute renal failure in a genetically predisposed patient with potential drug-drug interaction. International journal of clinical pharmacy 34(6), 825-7 (2012). doi:10.1007/s11096012-9717-0

[41] Araki, M., Maeda, M., Motojima, K.: Hydrophobic statins induce autophagy and cell death in human rhabdomyosarcoma cells by depleting geranylgeranyl diphosphate. European journal of pharmacology 674(2-3), 95-103 (2012). doi:10.1016/j.ejphar.2011.10.044

[42] Duke, J.D., Han, X., Wang, Z., Subhadarshini, A., Karnik, S.D., Li, X., Hall, S.D., Jin, Y., Callaghan, J.T., Overhage, M.J., Flockhart, D.A., Strother, R.M., Quinney, S.K., Li, L.: Literature based drug interaction prediction with clinical assessment using electronic medical records: novel myopathy associated drug interactions. PLoS computational biology 8(8), 1002614 (2012). doi:10.1371/journal.pcbi.1002614

[43] Wu, D., Wang, X., Zhou, R., Cederbaum, A.: CYP2E1 enhances ethanolinduced lipid accumulation but impairs autophagy in HepG2 E47 cells. Biochemical and biophysical research communications 402(1), 116-22 (2010). doi:10.1016/j.bbrc.2010.09.127

[44] Wu, D., Wang, X., Zhou, R., Yang, L., Cederbaum, A.I.: Alcohol steatosis and cytotoxicity: the role of cytochrome P4502E1 and autophagy. Free radical biology \& medicine 53(6), 1346-57 (2012). doi:10.1016/j.freeradbiomed.2012.07.005

[45] Aki, T., Funakoshi, T., Unuma, K., Uemura, K.: Impairment of autophagy: From hereditary disorder to drug intoxication. Toxicology 311(3), 205-15 (2013). doi:10.1016/j.tox.2013.07.001

[46] Rosenfeld, J.A., Ballif, B.C., Martin, D.M., Aylsworth, A.S., Bejjani, B.A., Torchia, B.S., Shaffer, L.G.: Clinical characterization of individuals with deletions of genes in holoprosencephaly pathways by aCGH refines the phenotypic spectrum of HPE. Human genetics 127(4), 421-40 (2010). doi:10.1007/s00439-009-0778-7 
[47] Mercier, S., David, V., Ratié, L., Gicquel, I., Odent, S., Dupé, V.: NODAL and SHH dose-dependent double inhibition promotes an HPE-like phenotype in chick embryos. Disease models \& mechanisms 6(2), 537-43 (2013). doi:10.1242/dmm.010132

[48] McKean, D.M., Niswander, L.: Defects in GPI biosynthesis perturb Cripto signaling during forebrain development in two new mouse models of holoprosencephaly. Biology open 1(9), 874-83 (2012). doi:10.1242/bio.20121982

[49] Shawlot, W., Wakamiya, M., Kwan, K.M., Kania, A., Jessell, T.M., Behringer, R.R.: Lim1 is required in both primitive streak-derived tissues and visceral endoderm for head formation in the mouse. Development (Cambridge, England) 126(22), 4925-32 (1999)

[50] Roy, A., Gonzalez-Gomez, M., Pierani, A., Meyer, G., Tole, S.: Lhx2 regulates the development of the forebrain hem system. Cerebral cortex, 1-12 (2013). doi:10.1093/cercor/bhs421

[51] Tu, C.-F., Yan, Y.-T., Wu, S.-Y., Djoko, B., Tsai, M.-T., Cheng, C.-J., Yang, R.-B.: Domain and functional analysis of a novel platelet-endothelial cell surface protein, SCUBE1. The Journal of biological chemistry 283(18), 12478-88 (2008). doi:10.1074/jbc.M705872200

[52] Ioannidis, J.P.A.: Why most published research findings are false. PLoS medicine 2(8), 124 (2005). doi:10.1371/journal.pmed.0020124 